\title{
„BITTE BETRACHTEN SIE MICH ALS EINEN TRAUM.“ SPRACHE UND IDENTITÄT IN HAMID SADRS GESPRÄCHSZETTEL AN DORA
}

\begin{abstract}
Wie Jacques Derrida u. a. in Die Einsprachigkeit des Anderen oder Die ursprüngliche Prothese zeigt, ist jeder Mensch immer schon mehrsprachig. Wenn wir diesen Zustand der Nicht-Einsprachigkeit als ein allgemein-menschliches Phänomen begreifen, und dazu eignet sich die sogenannte exophone Literatur besonders, dann hilft uns das zu verstehen, dass das Fremd-Sein eines/einer Fremden zu befragen immer zugleich bedeutet, auch unser eigenes Fremd-Sein zu befragen, dann wird die Frage nach dem Fremden zur Frage der eigenen Identität. Um dies zu zeigen, lese ich Hamid Sadrs Gesprächszettel an Dora (1994) parallel zu und mit Derridas Konzept von Ein- und Mehrsprachigkeit. In dem ,Roman“ erfindet der Erzähler entlang von Egodokumenten seine Wahrheit über Franz Kafkas Sterben und widerspricht damit vielen Wissenschaftler- und Biograph_innen, die den Tod Kafkas als Konsequenz seines Dichterseins, seiner Zerrissenheit etc. interpretiert haben. Was uns die Dichterfigur entfremdet, ist dabei die Sprache selbst und zwar gerade die anscheinend authentischen Berichte, die, in Kombination mit den unzuverlässigen Erzählerstimmen die Konstruktion der Figur Kafka offenlegen. Der Kafka des Textes - der als K., als Kafka, als kavka auftritt, der im Erzählen als Kafka gesetzt, erschrieben wird, und zwar in jenen Worten, die die seinen sind, die ihm also vermeintlich vorangehen und doch, zugleich, folgen, sich (auch) als Nach-Schreiben entpuppen - wird wieder aufgelöst, wird fremd. Die Verwirrung der Grenzen von Ursache und Wirkung, Vorher und Nachher, Realität und Fiktion, das Sein zwischen Leben und Tod lässt die Kafka/K.-Figur zum Wanderer, zur Figur des Sowohl-alsauch werden. Das Fremd-Sein Kafkas, die Exilsituation in der Abgeschiedenheit des Sanatoriums sind dabei nicht allein Parabeln auf die Situation exiliert Lebender, wie Sadrs persönliche Lebenssituation nahelegte, sie sind darüber hinaus eine Metapher für menschliche Identitätsfindung generell.
\end{abstract}

SCHLÜsSELwÖRTER: Jacques Derrida, Jacques Lacan, Paul de Man, Dekonstruktion, Autobiographie, Prosopopeia

\section{"BITTE BETRACHTEN SIE MICH ALS EINEN TRAUM". LANGUAGE AND IDENTITY IN HAMID SADRS GESPRÄCHSZETTEL AN DORA}

Abstract: According to Derridas Monolingualism of the Other, Or, The Prosthesis of Origin every person is always multilingual. If we understand this state of non-monolingualism as a general human phenomenon, which the so called 'exophone literature' particulary helps us to do, then we understand that to question a stranger's alienation always means to question our own alienation at the same time.

Peter Clar - Universität Wien, Wien, peter.clar@univie.ac.at 
Thus the question of the stranger becomes the question of one's own identity. In my article, I analyze this phenomenon by combining Hamid Sadr's 'novel' Gesprächzettel an Dora (1994) and Jacques Derrida's concept of mono- and multilingualism. In Hamid Sadr's text the narrator uses egodocuments to invent a truth about Franz Kafka's death which opposes many literary scholars and biographers who have interpreted Kafka's death as a consequence of his existence as a poet, his diremption etc. What alienates the literary figure named Kafka from us is the language itself, and first of all the combination of allegedly 'authentic' documents with the unreliable narrative voices, thus revealing the constructiveness of 'Kafka'. The main character Kafka - who within the text is named K., Kafka, kavka and who is introduced into the text by using his 'own' words, that supposedly precede him and yet, at the same time, succeed him - is deconstructed. Borders between cause and effect, before and after, reality and fiction, life and death become obsolete thus turning the Kafka/K. figure into a wanderer, a figure of 'both-and'. Kafka's situation as a stranger, as a kind of exile in the remoteness of the sanatorium, is not alone a parable on the situation of an exile, as Sadr's personal life may indicate. Moreover, as I will show, it is a metaphor for human identity in general.

Keywords: Jacques Derrida, Jacques Lacan, Paul de Man, deconstruction, autobiography, prosopopoeia

Lassen Sie mich, bevor ich zu Hamid Sadrs Gesprächszettel an Dora komme, zunächst den Begriff der ,Mehrsprachigkeit‘ umreißen (in dem ich jenen der Zweisprachigkeit enthalten sehe), lassen Sie mich vor allem darauf eingehen, warum ich diesen Begriff in meiner Forschung, die noch verhältnismäßig jung ist, im Plural, als ,Mehrsprachigkeiten“verwende. Zum einen spreche ich von Mehrsprachigkeiten, weil der Begriff in der Sprachwissenschaft, die sich innerhalb der Philologien natürlich besonders um ihn ,kümmert', schon ein vielschichtiger ist, schon allein deshalb weil, wie Georges Lüdi bereits 1996 feststellt, die engen Definitionen, nach denen sich „nur zwei oder mehrsprachig nennen darf, wer seine Sprachen in der frühesten Kindheit erworben hat und sie gleichermaßen perfekt schreibt und spricht", abgelöst worden seien durch jene, die jemanden als mehrsprachig definieren, die/der ,sich irgendwann in seinem Leben und Alltag regelmäßig zweier oder mehrerer Sprachvarietäten bedient und auch von der einen in die andere wechseln kann, [...] aber unabhängig von der Symmetrie der Sprachkompetenz, von den Erwerbsmodalitäten und von der Distanz zwischen den beteiligten Sprachen.“ (Lüdi 1996: 234) Mehrsprachig wären demnach auch alle, die beispielsweise zwischen einem Dialekt und der Hochsprache hin und her wechseln können. Meiner Auswahl von Autor_innen, mit denen ich mich im Rahmen der Frage nach der Mehrsprachigkeit beschäftige, liegt dann doch eine etwas engere Definition zu Grunde - eine Definition, die aber, wie alle Grenzziehungen, willkürlich, hinterfragbar, in sich selbst nicht schlüssig ist, doch dies zu explizieren, führte in diesem Rahmen zu weit. Von Lüdi ausgehend zählt Chiara Messina in ihrem Überblicksartikel zur Forschungslage der Mehrsprachigkeitsforschung folgende Mehrsprachigkeiten auf, die unterschieden würden, auch wenn sie niemals vollständig voneinander zu trennen seien (vgl. Messina 2010: 112): 
- Individuelle Zwei- bzw. Mehrsprachigkeit,

- Ungesteuerter Bilinguismus: Simultaner Bilinguismus,

- Gesteuerter oder ungesteuerter Bilinguismus: Sukzessiver Bilinguismus; später, sukzessiver Bilinguismus; symmetrischer vs. asymmetrischer Bilinguismus; bilinguale oder bidialektale Mehrsprachigkeit,

- Soziale Mehrsprachigkeit (Diglossie),

- Territoriale Mehrsprachigkeit,

- Institutionelle Mehrsprachigkeit.

Neben diesem sprachwissenschaftlichen Zugang ist aber zu fragen, was denn ,mehrsprachige Literatur' überhaupt bedeuten kann. Ist denn jeder Text eines/einer, wie auch immer definierten, mehrsprachigen Autors/Autorin mehrsprachige Literatur? Zählen nur jene Texte zur mehrsprachigen Literatur, die Mehrsprachigkeit inhaltlich thematisieren? Oder muss ein mehrsprachiger Text tatsächlich mehrsprachig sein - und wenn ja, in welchem Ausmaß? Reichen einzelne Zitate beispielsweise englischer Liedtexte? Oder muss es ,mehr' sein - und wenn ja, wieviel? Schon die Strategien des Einarbeitens von Mehrsprachigkeit als zunächst formalem Mittel (dass Form und Inhalt natürlich niemals voneinander zu trennen sind, ist klar) sind vielfältig, wie Dirk Skiba zeigt (vgl. Skiba 2010). In seiner Untersuchung deutscher Migrationsliteratur unterscheidet er zunächst jene Autor_innen, die einen vollständigen Sprachwechsel vollzogen haben, jene, die sich einem Sprachwechsel vollständig verweigern, und jene, die einen partiellen Sprachwechsel vollziehen. Bei letzterer Gruppe gäbe es diejenigen, die je nach Gattung die Sprache wechseln, und diejenigen, die innerhalb eines Textes mit Mehrsprachigkeit arbeiteten. Die Strategien reichen dabei vom völlig unkommentierten Einbauen fremdsprachiger Elemente in die Grundsprache des Textes, über kommentierte Formen (mittels Fußnoten, Kontext, Übersetzungen im Text etc.) bis hin zum Erfinden von Kunstsprachen wie beispielsweise dem ,Denglisch “ von Gayle Tuft. Hamid Sadrs Gesprächszettel an Dora (1994) müsste man nach dieser Einteilung als jenen Text bezeichnen, mit dem der 1946 in Teheran geborene Autor den vollständigen Sprachwechsel vollzog. Bis dahin hatte der 1967, vorerst wegen des Studiums, nach Österreich gekommene Sadr in seiner Muttersprache geschrieben. Nachdem der Schriftstellerverband, dessen Mitglied er war, vom Schahregime verboten worden war, beschloss er, in Europa zu bleiben, zunächst in Wien, dann nach der islamischen Revolution in Paris, seit 1991 wieder in Wien. Seit 1994 schreibt er also, glaubt man den wenigen Quellen, die man zu Sadr findet, fast ausschließlich in deutscher Sprache (vgl. u. a. Vlasta 2015; Internetseite des Autors).

Was mich in Bezug auf ,Mehrsprachigkeiten " aber besonders interessiert - und damit komme ich gleich weniger zur Analyse von Gesprächszettel an Dora als vielmehr zu einer Analyse mit/entlang dieses Textes - ist die, den Menschen immer schon eingeschriebene Mehrsprachigkeit im Sinne von Derridas „Ja, ich habe nur eine Sprache, und die ist nicht die meinige" (Derrida 2003: 13). Was Derrida mit dieser und ähnlichen Antinomien („1. Man spricht immer nur eine einzige Sprache. 2. Man spricht niemals 
eine einzige Sprache.“ (Derrida 2003: 19)) in Die Einsprachigkeit des Anderen oder die ursprüngliche Prothese beschreibt bzw. umkreist, ist das Verständnis der Verfasstheit des Individuums als grundsätzlich gespaltenes. „Ja, ich habe nur eine Sprache, und die ist nicht die meinige" impliziert, dass wir niemals nur ein Ich, dass wir viele, dass wir ,multipliiert' (vgl. Lücke 2007: 62) und uns daher immer auch fremd sind, ganz im Sinne von Kristevas „Fremd sind wir uns selbst“ (Kristeva 1990). Was uns vor Augen geführt wird, ist die (immer prozesshafte) Erkenntnis, nicht das (fixierte) Wissen, dass im Eigenen immer schon das Fremde eingeschrieben ist, wie im Fremden das Eigene, dass man sich eine Sprache (und damit eine Identität, sei es eine nationale oder individuelle) niemals (vollständig) an-eignen kann. Wenn wir aber den Zustand der Nicht-Einsprachigkeit als ein allgemein-menschliches Phänomen begreifen, und dazu eignet sich die sogenannte exophone Literatur vielfach besonders, nicht, weil das Phänomen dort exklusiv, aber oftmals deutlicher verhandelt wird, dann, so meine These, hilft uns das zu verstehen, dass die Situation/das Leben/das Fremd-Sein eines/einer (auf Grund welcher Ausschlüsse auch immer) Fremden zu befragen immer zugleich bedeutet, auch unsere Situation/unser Leben/unser Fremd-Sein zu befragen - vielleicht etwas, was gerade auch heute, als Empfehlung sowohl an die politischen Player als auch an jede/n einzelne/n von uns gelesen werden sollte. Es geht mir also im Folgenden nicht darum, eine besondere, an die biographischen Hintergründe gekoppelte Schreibweise Hamid Sadrs festzustellen (wie sollte das auch gelingen?), sondern seinen Text als ein komplexes Spiel der Verknüpfung von Sprache/Schreiben und Identitätsentwürfen zu lesen, welches in eine Auflösung feststehender Gewissheiten mündet - und somit prototypisch für die Frage nach der Identitätskonstruktion jeder/jedes Einzelnen wird.

\section{2}

Am Ende von Hamid Sadrs ,Roman“ (vgl. Sadr 1994: 215ff.), welches, wie ein Epilog, dem Leben/dem Sterben Kafkas folgt, wie im Übrigen auch der Prolog dem Leben/dem Sterben Kafkas folgt, obwohl er dem Leben/dem Sterben Kafkas vorangeht, wie auch der Epilog dem Leben/dem Sterben Kafkas vorangeht, zumindest jener Version des Lebens/des Sterbens vorangeht, die dieses Buch erzählt (und über etwas anderes kann ich nicht schreiben, wie ich immer nur über über Kafka Geschriebenes schreiben kann, nie über sein Leben/Sterben), am Ende also jenes ,Romans ${ }^{6}$, dessen Klammer bereits die Chronologie auflöst, Vor- und Nachgängigkeit(en) verwirrt, liest Max Brod in seinem Gedächtnisheft, wie am Anfang der Ich-Erzähler (oder doch der Autor?; immerhin haben wir es nach dem Prolog mit einem personalen Erzähler zu tun, der, manchmal unterstützt von den Bäumen, den Wänden, den Brettern des Balkons und vor allem einer Dohle, von den letzten Wochen Kafkas berichtet, die letzten Wochen Kafkas erschreibt) in Max Brods Buch über Kafka liest (vgl. Sadr 1994: 5), doch das nur (wie) nebenbei. Die Stelle, die Max Brod in Vorbereitung auf seine Trauerrede in 
seinem Gedächtnisheft liest (damit zum Leser seiner eigenen Zeilen, zum ,Autor-Leser', zu, um mit Kristeva zu sprechen, seinem eigenen Gesprächspartner ${ }^{1}$ werdend, zum Leser und Autor zugleich werdend, wie er zugleich fiktive und reale, lebende und tote Figur ist) und die den Text zu ungefähr jenem Zeitpunkt enden lässt, mit dem er eingesetzt hat (einsetzen wird?), lautet: „Als er einmal nachmittags zu mir kam, weckte er meinen Vater, der auf dem Sofa schlief. Zur Beschwichtigung die Arme hebend und leise auf den Fußspitzen durchs Zimmer gehend, sagte er: ,Bitte betrachten Sie mich als einen Traum. “" (Sadr 1994: 217)

Wer aber ist es, der uns hier als vermeintlicher Traum entgegentritt, der sich mit den Worten „Bitte betrachten Sie mich als einen Traum“ als unecht, irreal, nicht existent setzt und damit gerade seine Echtheit, sein real-Sein, seine Existenz bekräftigt, mehr noch, sich selbst als echt, real, existierend erschreibt? Die Antwort ,Franz Kafka scheint einfach, schiene wohl auch dann einfach, wenn man nicht am Cover des Buchs ein Foto des berühmten Schriftstellers sehen könnte, wenn auf der Buchrückseite nicht sein Name abgedruckt wäre, kommt doch bereits im nur wenige Zeilen umfassenden Prolog der Name ,Kafka ' fünf Mal vor. Und auch wenn der Vorname fehlt, so fehlt es nicht an zahlreichen biographischen Hinweisen, Barthes paraphrasierend könnte man von Biographemen sprechen, ${ }^{2}$ dem Todestag (3.6.1924), dem Todesort (Kierling) oder Namen aus dem Umfeld Franz Kafkas (Max Brod, Dora, Robert Klopstock, Werfel) (vgl. Sadr 1994: 5). Und doch, so einfach ist es nicht, ganz einfach, weil es nie einfach ist, weil, um Ingeborg Bachmann abzuwandeln,

[...] sich beweisen läßt, daß es Kafka gibt [bzw. gab], man [ihn] aber mit einem Wort nicht treffen kann, weil Kafka hier auf dem Papier ist [...] und hier also nicht Kafka sein kann, weil hier nur Worte sind, die anspielen und insistieren auf etwas, das es gibt, und auf anderes, das es nicht gibt [...] (Bachmann 1993: 345).

Was man aber tun kann, und was Hamid Sadrs ,Roman“ (der ebenso eine Biographie, eine Dokumentation, ein kritischer Kommentar zur Rezeption Kafkas, eine Anamnese seiner Krankengeschichte etc. ist) macht, ist zu zeigen, dass man Kafka nicht mit einem Wort (aber auch nicht mit hunderten, tausenden Worten) treffen kann, dass man im Sprechen über eine Person (in diesem Fall Kafka) sich dieser immer nur annähern kann, sie, indem man über sie spricht (oder sie anspricht), immer auch erst setzt, konstruiert, dass, indem man jemanden beschreibt (oder anspricht), diese/n immer auch erschreibt, umschreibt, neu schreibt. Dabei aber verfehlt man diese Person immer schon, weil das Gegenüber zwangsläufig verfehlt wird, weil es im Moment des beschrieben-Werdens,

${ }^{1}$ „The writer's interlocutor, then, is the writer himself, but as reader of another text. The one who writes is the same as the one who reads. Since his interlocutor is a text, he himself is no more than a text re-reading itself as it rewrites itself. The dialogical structure, therefore, appears only in the light of the text elaborating itself as ambivalent in relation to another text.“ (Kristeva 1986: 56f.)

${ }^{2}$ Vgl. Barthes (1986). 
des angesprochen-Werdens (momenthaft) erst zur Existenz gebracht wird. Diesen Prozess bezeichnet Paul de Man, und in seinem Gefolge Theoretiker_innen wie Bettine Menke, Anna Babka, Werner Hamacher etc., als Prosopopeia, als die

\begin{abstract}
Fiktion der Apostrophierung einer abwesenden oder stimmlosen Entität, wodurch die Möglichkeit einer Antwort gesetzt und der Entität die Macht der Rede zugesprochen wird. Eine Stimme setzt einen Mund voraus, ein Auge und letztlich ein Gesicht, eine Kette, die sich in der Etymologie des Namens der Trope manifestiert: prosopon poien, eine Maske oder ein Gesicht (prosopon) geben. Die Prosopopöie ist die Trope der Autobiographie, durch die jemandes Name, wie in dem Gedicht Miltons, so verstehbar und erinnerbar wird wie ein Gesicht. (de Man 1993: 140)
\end{abstract}

Die Gespaltenheit zwischen Be- und Erschreiben, zwischen Erkennen und Verfehlen, zwischen ein Gesicht geben und ein Gesicht erinnern verweist dabei auch zurück auf die strukturelle Gespaltenheit jeder Identität, auch der eigenen, so wie sie schon Jacques Lacan in seinem vielzitierten Aufsatz Das Spiegelstadium als Bildner der Ichfunktion konstatiert. Denn das Kind, welches sich zum ersten Mal als vollständig wahrnimmt, kann dies vorerst nur durch den Blick in den Spiegel tun, die Erfahrung als vollständiges Subjekt ist aber nicht durch seine motorischen Fähigkeiten erfahrbar, sondern vom ,Anderen', vom (imaginären) Gegenüber im Spiegel abhängig - einem Gegenüber, das wiederum von dem Kind vor dem Spiegel abhängig ist. Das Individuum ist also immer schon ,Dividuum', das Erkennen als kohärentes Lebewesen immer gekoppelt an ein Verkennen, das, „was der Mensch als eigenes annimmt, seine Selbstgewißheit, ist ihm fundamental Fremdes.“(Bittel: o. J., o. A.)

Auf diese strukturelle Verbundenheit von Fremdheit und Selbstheit gerade im vermeintlich so individuellen Prozess der Ich-Werdung rekurriert auch Jacques Derrida, wenn er schreibt: „,W]as ist Selbstheit? Diese beschränkt sich nicht auf die abstrakte Fähigkeit, ,ich` zu sagen, der sie immer schon vorausgegangen sein wird. Sie bedeutet vielleicht in erster Linie die Macht eines ,ich kann', das ursprünglicher ist als das ,ich“" (Derrida 2003: 30). Dieses ,Ich-(kann)-Sagen“ ist dabei immer schon abhängig von einer dem Ich vorhergehenden Sprache, ,das Ich der sogenannten autobiographischen Anamnese, das Ich-mich des ich erinnere mich [...] produziert und äußert sich“, so Derrida, ,je nach Sprache verschieden [...], es ist also nicht unabhängig von der Sprache im allgemeinen“ (Derrida 2003: 52). Die Sprache aber ist nie die eigene, jeder, nicht nur der/die Exilant_in, Migrant_in, ist der vermeintlich eigenen Sprache immer schon entfremdet, denn die Möglichkeit Ich zu sagen setzt ein Ich voraus, welches spricht, welches aber bereits gesagt worden sein muss. Das Ich (und seine Sprache) ist immer bereits nur nachgesprochen, gründet immer in einer ihm vorausgehenden Sprache, der Sprache des/der Anderen, ist immer schon Wiederholung, dabei aber eine „Imitation ohne Original“ (Butler 1991: 203). Denn wie das Ich nicht ohne Vor-Sprache existieren kann, kann diese nicht ohne das Ich existieren, die Sprache ist dem Ich ebenso vor- wie nachgängig, sie erzeugt das Ich ebenso, wie dieses die Sprache erzeugt - eine Verwirrung von Zeitlichkeiten, von 
chronologischen Abläufen ähnlich jener, die Gesprächszettel an Dora erzeugt, wie ich am Beginn meiner Ausführungen, zu der diese Zeilen immer noch gehören, gezeigt habe oder zeige oder zeigen werde.

Es ist diese Verwirrung der Grenzen von Vorher und Nachher, von Ursache und Wirkung, Realität und Fiktion, das Sein zwischen Leben und Tod (,denn jetzt bin ich schon Bürger in dieser anderen Welt" (Sadr 1994: 90)), die die Kafka-Figur in Sadrs Text zur Figur des Sowohl-als-Auch und damit zur, wenn man so will, Metapher menschlicher Identität werden lässt. Trotz der im Prolog skizzierten ,autobiographischen Fakten“, die allerdings Zitate aus einem Buch sind („In Max Brods Buch über Kafka las ich [...]“(Sadr 1994: 5)), die also Schrift/Sprache sind und damit nicht vertrauenswürdig (,ich vertraue Worten und Briefen nicht“, zitiert der Text aus einem Brief Kafkas an Brod), ist ,Kafka' von Beginn an brüchig, nicht greifbar, und so lautet der erste Absatz nach dem Prolog: „Kafka war nicht geneigt zu sterben, nehme ich an. Aber um das zu belegen, werden drei Zimmer, eine Dohle und Wien - als Stadt - nötig sein.“ (Sadr 1994: 7) Schon im ersten Halbsatz opponiert der Text „gegen die hagiographische Gedankenlosigkeit, mit der ,frühe Dichtertode' raunend ausgelegt und mystifiziert werden" (Wallmann 1994), wehrt er sich gegen die Überhöhung des Todes Kafkas durch die Fachliteratur ,als tragisches Ende eines konsequent der Literatur gewidmeten und sich dafür selbst verzehrenden Lebens“ (Hoenig, o. J.), ohne aber eine eigene Wahrheit gegen diese zu stellen, wie das ,nehme ich an“ deutlich macht, ebenso wie die unzuverlässigen Zeugen, die ins Feld geführt werden, drei Zimmer, Wien, eine Dohle; genannt werden zudem u. a. „Die Gesprächsblätter (auch Gesprächszettel genannt)“, mit deren „Hilfe [...] sich K. verständigen [mußte], als ihm die Schweigekur verordnet worden war" (Sadr 1994: 10), die Blumen, aber auch einige Personen wie z. B. der „Wieshaider Lorenz (genannt Wieshaider Lenzl)“, über den es aber kurz darauf heißt, er komme ,als Zeuge nicht in Frage, weil er schon vor Jahren, wenn nicht im Krieg, dann zu Hause, gestorben“ (Sadr 1994: 11) sei. Wie die Kafka-Figur ungreifbar ist, sind es die Zeug_innen, ist es der Wieshaider Lenzl, der, obwohl tot, als Zeuge aufgerufen wird, sind es die Gesprächszettel, im Text akribisch mit Nachweis versehen, die „uns bei manchen Fragen weiterhelfen“ können, von denen wir aber nicht wissen „ob es sich [...] um alle diese Zettel handelt" (Sadr 1994: 10). Zudem durchziehen den Text auch immer wieder Zitate, deren Herkunft nicht nachgewiesen ist, die vielleicht oder wahrscheinlich erfunden sind. Gerade die Mischung aus historisch verbrieften Dokumenten (den Gesprächszetteln, den genauen Wetterberichten der Hohen Warte, der exakten, zum Teil mit wissenschaftlichen Begriffen versehenen Krankengeschichte etc.) mit den offensichtlichen Erfindungen ist es, die uns alle Figuren, allen voran Kafka, entfremdet, die jenen Kafka uns entfremdet, der uns von der Rezeption vorgeführt 
wurde (jenes, um mit Scheer zu sprechen, „kafkaesk anmutende[] Monument[], das den Menschen Kafka verdeckt" (Scheer 1994: 21)) - ohne dass der Text dabei eine andere Wahrheit präsentierte:

Es läuft zur Sicherheit, nicht nur um mich zu behüten, meine Sprache neben mir her und kontrolliert, ob ich es auch richtig mache, ob ich es auch richtig falsch mache, die Wirklichkeit zu beschreiben, denn sie muß immer falsch beschrieben werden, sie kann nicht anders, aber so falsch, daß jeder, der sie liest oder hört, ihre Falschheit sofort bemerkt[,] (Jelinek 2004: 229f.)

schreibt Elfriede Jelinek, und so beschreibt Gesprächszettel an Dora Kafka immer wieder neu und anders, widersprüchlich, kurz: so falsch, dass man die „Falschheit sofort bemerkt". Und doch liegt gerade im Ausstellen des Verfehlens dessen, was der Text auf den ersten Blick zu tun vorgibt, nämlich das Leben/Sterben des Franz Kafka nachzuzeichnen, zu dokumentieren, zu beschreiben, zwar keine größere Wahrheit, aber vielleicht so etwas wie eine größere Wahrscheinlichkeit: „Wahrscheinlichkeit, aber keine Wahrheit: Freischeinlichkeit, aber keine Freiheit - diese beiden Früchte sind es, derentwegen der Baum der Erkenntnis nicht mit dem Baum des Lebens verwechselt werden kann“ (Nietzsche 2012: 8), schreibt Nietzsche, und bei Sadr heißt es: „Nicht jeder kann die Wahrheit sehen, aber sein [...]“ (Sadr 1994: 77).

„Nicht jeder kann die Wahrheit sehen, aber sein, denkt sich die Dohle“, denkt sich die ,kavka', die tschechische Dohle und am Ende meiner Ausführungen möchte ich, noch kursorischer als bisher schon und ohne eine Conclusio zu versprechen, kurz auf diese ,Kafka-Figur` eingehen. In Die Postkarte fügt Jacques Derrida* seinem Namen am Ende der Einleitung einen Asterisk hinzu, weil er „ohne Zweifel mehrere“ sei und „nicht so allein, wie ich es bisweilen sage“(Derrida 1982: 11). Damit hinterfragt er (wie mit dem gesamten Text, wie auch in anderen Texten) die Vorstellung, der Name bürge oder lege Zeugnis ab für eine hinter diesem Namen feststehende Identität. In Gesprächszettel an Dora tritt nun die tschechische Dohle, die ,kavka', Kafka gegenüber (und fungiert damit, das aber nur nebenbei, weniger als Zeugin denn als Kommentatorin), um ihm immer wieder zu widersprechen, seinem (oder auch Doras) Selbstbetrug über den tatsächlichen Gesundheitszustand entgegenzuwirken. Nicht nur der Name ,kavka', der ja kein Name, sondern vielmehr eine Gattungsbezeichnung ist, der aber doch auch den Namen ,Kafka' wiederholt, weist dabei darauf hin, dass die ,kavka' ,Kafka' ist, besser, eine Version, eine Möglichkeit von ihm ist, wenn sie zum Beispiel mit profunden Kenntnissen der Kafka'schen Tagebücher argumentiert: „Kafka staunt, wie kann die Dohle wissen, was er vor Jahren an einem Morgen dachte und in das Tagebuch schrieb.“(Sadr 1994: 80) Die Kavka belehrt Kafka mit einem Zitat von Kafka, ,Kafka“ 
ist also zumindest dreifach und ist noch mehr, wird an anderer Stelle als K. bezeichnet, und damit auch zur Figur der ,eigenen“ Texte, Kafka selbst schreibt sich als K. in sein Leben/in den Roman Sadrs ein (chronologische Unmöglichkeit). Und apropos literarische Figur, unübersehbar sind auch die Parallelen zwischen dem, ob der großen Schmerzen beim Schlucken, hungernden Kafka und der Figur des Hungerkünstlers, ${ }^{3}$ dessen Fahnen Kafka permanent korrigiert. Die Figur Kafkas ist gespalten, vervielfacht, ,multipliiert‘, wie jede Identität gespalten, vervielfacht, ,multipliiert‘, wie wir alle gespalten, vervielfacht, ,multipliiert' und uns daher immer auch fremd sind, ganz im Sinne von Kristevas „Fremd sind wir uns selbst“. Was uns vor Augen geführt wird, ist die (immer prozesshafte) Erkenntnis, nicht das (fixierte) Wissen, dass im Eigenen immer schon das Fremde eingeschrieben ist, wie im Fremden das Eigene, dass man sich eine Sprache (und damit eine Identität, sei es eine nationale oder individuelle) niemals (vollständig) an-eignen kann. Wenn wir aber den Zustand der Nicht-Einsprachigkeit als ein allgemein-menschliches Phänomen begreifen - und dazu eignet sich die sogenannte exophone Literatur vielfach besonders, nicht, weil das Phänomen dort exklusiv, aber oftmals deutlicher verhandelt wird - dann, so meine These, hilft uns das zu verstehen, dass die Situation/das Leben/das Fremd-Sein eines/einer (auf Grund welcher Ausschlüsse auch immer) Fremden zu befragen immer zugleich bedeutet, auch unsere Situation/unser Leben/unser Fremd-Sein zu befragen - vielleicht etwas, was gerade auch heute, als Empfehlung sowohl an die politischen Player als auch an jede/n einzelne/n von uns gelesen werden sollte. Aber ich glaube, soweit waren wir schon.

\section{Literatur}

Bachmann, I. (1993). Der Fall Franza. In I. Bachmann, Werke 3 (S. 339-482). München: Piper.

Barthes, R. (1986). Sade Fourier Loyola. Frankfurt a. M.: Suhrkamp.

Bittel, J. (o. J.). ,Sterben, Schlafen - Schlafen, Träumen vielleicht. 'Zum Spiegelstadium bei Lacan Teil I. Abgerufen am 25.10.2019 von http://www.salmoxisbote.de/Bote08/Bittel.htm.

Butler, J. (1991). Das Unbehagen der Geschlechter (K. Menke, Übers.). Frankfurt a. M.: Suhrkamp.

Derrida, J. (1982). Die Postkarte von Sokrates bis an Freud und jenseits. 1. Lieferung: Envois/Sendungen (H.-J. Metzger, Übers.). Berlin: Brinkmann \& Bose.

Derrida, J. (2003). Die Einsprachigkeit des Anderen oder die ursprüngliche Prothese (M. Wetzler, Übers.). München: Wilhelm Fink.

Hoenig, M. (o. J.). Kafkas letzte Lebenswochen in einem Roman dargestellt [Besprechung von Hamid Sadrs Gesprächszettel an Dora]. Abgerufen am 25.10.2019 von http://members.aon.at/hamidsadr/ sadr/roman2.html.

Internetseite des Autors. Abgerufen am 25.10.2019 von www.hamidsadr.com.

Jelinek, E. (2004). Im Abseits. In P. Janke (Hrsg.), Nobelpreis Elfriede Jelinek (S. 227-238). Wien: Praesens. Kafka, F. (1996). Ein Hungerkünstler. In F. Kafka, Drucke zu Lebzeiten. Hrsg. von W. Kittler, H.-G. Koch \& G. Neumann (S. 315-377). Frankfurt a. M.: Fischer.

${ }^{3}$ Vgl. Kafka (1996). 
Kristeva, J. (1986). Word, Dialogue and Novel. In T. Moi (Hrsg.), The Kristeva Reader (S. 34-61). New York: Columbia University Press.

Kristeva, J. (1990). Fremde sind wir uns selbst (X. Rajewsky, Übers.). Frankfurt a. M.: Suhrkamp.

Lücke, B. (2007). Elfriede Jelineks ästhetische Verfahren und das Theater der Dekonstruktion. Von Bambiland/Babel über Parsifal (Laß o Welt o Schreck laß nach) (für Christoph Schlingensiefs ,Area 7“) zum ,Königinnendrama' Ulrike Maria Stuart. In P. Janke \& Team (Hrsg.), Elfriede Jelinek: „ICH WILL KEIN THEATER“. Mediale Überschreitungen (S. 61-83). Wien: Praesens.

Ludi, G. (1996). Mehrsprachigkeit. In H. Goebl, P.H. Nelde, S. Zdeněk \& W. Wolck (Hrsg.), Kontaktlinguistik. Ein internationales Handbuch zeitgenössischer Forschung. 1. Halbband (S. 233-245). Berlin: de Gruyter.

de Man, P. (1993). Autobiographie als Maskenspiel (1979). In P. de Man, Die Ideologie des Ästhetischen (J. Blasius, Übers.) (S. 131-146). Frankfurt a. M.: Suhrkamp.

Messina, C. (2010). Zweisprachigkeit vs. Mehrsprachigkeit. In M. Bürger-Koftis, H. Schweiger \& S. Vlasta (Hrsg.), Polyphonie. Mehrsprachigkeit und literarische Kreativität (S. 107-133). Wien: Praesens.

Nietzsche, F. (2012). Der Wanderer und sein Schatten. Zweiter und letzter Nachtrag zum Buch der freien Geister. Köln: Anaconda.

Sadr, H. (1994). Gesprächszettel an Dora. Wien: Deuticke.

Scheer, U. (25. Juni 1994). Demontage einer verwirrten Dohle. Die Welt, 21.

Skiba, D. (2010). Formen literarischer Mehrsprachigkeit in der Migrationsliteratur. In M. Bürger-Koftis, H. Schweiger \& S. Vlasta (Hrsg.), Polyphonie. Mehrsprachigkeit und literarische Kreativität (S. 323334). Wien: Praesens.

Vlasta, S. (2015). Contemporary Migration Literature in German and English. Leiden: Brill.

Wallmann, H. (27. April 1994). Bitte, betrachten Sie mich als einen Traum. Hamid Sadr macht uns Kafka wieder fremd: ,Gesprächszettel an Dora'. Süddeutsche Zeitung, 15. 